\title{
Re: Varicocelectomy to "Upgrade” Semen Quality to Allow Couples to Use Less Invasive Forms of Assisted Reproductive Technology
}

\author{
Samplaski MK'1, Lo KC², Grober ED², Zini $A^{3}$, Jarvi KA ${ }^{4}$ \\ ${ }^{1}$ University of Southern California, Institute of Urology, Los Angeles, California \\ ${ }^{2}$ Mount Sinai Hospital, Department of Surgery, Division of Urology, Toronto, Ontario, Canada \\ ${ }^{3}$ McGill University, Department of Surgery Montreal, Division of Urology, Quebec, Canada \\ ${ }^{4}$ Mount Sinai Hospital, Department of Surgery, Division of Urology, Toronto, Canada; University of Toronto, Institute of Medical Sciences, Toronto, \\ Canada
}

Fertil Steril 2017;108:609-612. doi: 10.1016/j.fertnstert.2017.07.017.

\section{EDITORIAL COMMENT}

Varicocele is one of the most commonly seen and correctable anatomical pathology in infertile men. Although varicocele repair results in improvement of semen quality in most infertile men, the degree of improvement in semen parameters is not well studied. The authors aimed in this study to determine the magnitude of improvement in semen parameters and the fraction that have improvements after a varicocelectomy in order to pave the way for less invasive techniques for couples needing assisted reproductive techniques. The main outcome measurement is total motile sperm count (TMSC) before and after the varicocele repair. For natural pregnancy, more than 9 million, for intrauterine insemination (IUI)-5-9 million and for in vitro fertilization (IVF), less than 5 million TMSC are considered the cut off points. A total of 373 men underwent varicocele repair and the mean TMSC increased from 18 to 46 million which is statistically significant. The most pronounced increase was in the baseline TMSC less than 5 million group and 58\% of men were upgraded from IVF candidacy to IUI of natural pregnancy. As a conclusion, the authors emphasized that even for low TMSC, a varicocelectomy may reduce the need for IVF and invasive ART techniques.

Emre Bakırcıoğlu, MD

๑Copyright 2018 by the Association of Urological Surgery / Journal of Urological Surgery published by Galenos Publishing House. 